\title{
Distribution of uridine diphosphate glucuronosyltransferase 1A polymorphisms and their role in irinotecan-induced toxicity in patients with cancer
}

\author{
YANG WANG $^{1}$, CUIHUA YI ${ }^{1}$, YAWEI WANG ${ }^{1}$, HUI LI $^{2}$, BEI LI ${ }^{1}$, \\ DAN WANG $^{1}$, JINTONG DU ${ }^{3}$, LIAN LIU ${ }^{1}$ and XIUWEN WANG ${ }^{1}$ \\ ${ }^{1}$ Department of Chemotherapy, Cancer Center, Qilu Hospital of Shandong University, Jinan, Shandong 250012; Departments \\ of ${ }^{2}$ Pharmacy and ${ }^{3}$ Medical Oncology, Shandong Cancer Hospital and Institute, Jinan, Shandong 250117, P.R. China
}

Received October 28, 2015; Accepted April 13, 2017

DOI: $10.3892 / \mathrm{ol} .2017 .6933$

\begin{abstract}
Uridine diphosphate glucuronosyltransferase 1A (UGT1A1), which affects irinotecan metabolism, has been associated with severe adverse reactions in patients with cancer treated with irinotecan. However, neither large-scale analysis of the distribution of UGT1A1 polymorphisms, nor standardized assessment of how UGT1A1 polymorphisms affect irinotecan treatment has been performed in China. The aim of the present study was to investigate the distribution of UGT1A1 polymorphisms ( 28 and *6) in 2,093 Chinese patients with cancer who were treated with irinotecan from more than 15 hospitals in Shandong, to examine how the coexistence of UGT1A1* 6 and UGT1A1*28 alleles may be able to predict toxicities induced by irinotecan in 105 of the patients, and to search for other relevant risk factors. The distribution of the genotypes was as follows: $\mathrm{TA}_{6} / \mathrm{TA}_{6}(1,601,76.5 \%), \mathrm{TA}_{6} / \mathrm{TA}_{7}(463,22.1 \%)$ and $\mathrm{TA}_{7} / \mathrm{TA}_{7}(29,1.4 \%)$ for UGT1A1 $28(\mathrm{n}=2,093)$; and G/G $(286$, $66.4 \%), \mathrm{G} / \mathrm{A}(124,28.8 \%)$ and A/A $(21,4.9 \%)$ for UGT1A1*6 $(n=431)$. The most frequent severe hematological toxicity was neutropenia, and the predominant non-hematological toxicities were diarrhea and cholinergic syndrome. In toxicity comparisons, grade 3-4 leukopenia and neutropenia were significantly higher in $\mathrm{TA}_{6} / \mathrm{TA}_{7}$ compared with $\mathrm{TA}_{6} / \mathrm{TA}_{6}(\mathrm{P}<0.05)$. The UGT1A1* 6 polymorphism was associated with a higher risk of severe diarrhea and total adverse drug reactions $(\mathrm{P}<0.05)$. Logistic regression showed that the UGT1A1* 6 genotype was an independent predictor of severe diarrhea. These findings suggested that the UGT1A1 28 and UGT1A1* 6 genotypes may be associated with irinotecan-induced severe toxicity, and clarified the clinical importance of UGT1A1 polymorphisms,
\end{abstract}

Correspondence to: Professor Xiuwen Wang, Department of Chemotherapy, Cancer Center, Qilu Hospital of Shandong University, 107 West Wenhua Road, Jinan, Shandong 250012, P.R. China

E-mail: xiuwenwang12@sdu.edu.cn

Key words: uridine diphosphate glucuronosyltransferase 1A, irinotecan, toxicity, polymorphism particularly $\mathrm{UGT} 1 \mathrm{~A} 1^{*} 6$, regarding irinotecan therapy in Chinese patients.

\section{Introduction}

Irinotecan, a camptothecin analogue that inhibits topoisomerase $\mathrm{I}$, is a cytotoxic agent used widely for the treatment of solid tumors, including colorectal (1,2), lung (3), ovary (4) and gastric (5) cancer. However, treatment with this drug frequently results in delayed-diarrhea and severe hematological toxicities, including neutropenia and leukopenia, which can markedly impact the course of treatment and the quality of life of patients, and even limit its clinical application (6). Therefore, tolerable and efficient individualized salvage treatment regimens for irinotecan-treated cancer patients are required.

One of the isoforms of uridine diphosphate glucuronosyl transferase (UGT), UGT1A1, is the main enzyme involved in the metabolism of irinotecan, which converts the active metabolite of irinotecan (SN-38) to an inactive glucuronide (SN-38G) (7). Inter-individual variability in the pharmacokinetics of SN-38 appears to be associated with severe neutropenia and delayed-diarrhea (8). UGT1A1*28 [-53(TA) 6>7] leads to decreased glucuronidation of SN-38; thus, genotyping of the UGT1A1*28 allele may help to predict patient vulnerability to irinotecan-associated toxicity in early studies $(9,10)$. These findings led the U.S. Food and Drug Administration to require that gene-associated information is added to drug product labels in 2005 (11). However, subsequent studies demonstrated several inconsistencies $(12,13)$, considering that dose-limiting neutropenia and diarrhea were dependent on numerous known and unknown factors.

Although the UGT1A $1^{*} 28$ allele is considered to be an important predictor of irinotecan-associated toxicity, differences in ethnicity have also been reported $(14,15)$. The UGT1A1*28 allele frequency in Asian individuals is reduced compared with that in Caucasian individuals, while severe hematological toxicity is associated with polymorphisms in UGT1A1*6 in the Asian population (16). Several previous Asian studies revealed that severe adverse events were associated with the homozygosity of the UGT1A1*6 allele (17-19). Thus, the UGT1A1*6 genotype appears to be another 
important predictor of irinotecan-induced adverse events. However, neither large-scale analysis of the distribution of UGT1A1 polymorphisms, nor standardized assessment of how UGT1A1 polymorphisms effect irinotecan treatment has been performed in China.

To understand the clinical significance of these variants, particularly the more frequent variant of UGT1A1" 6 , the frequencies of UGT1A1 variants were examined in 2,093 Chinese patients from 15 hospitals in Shandong province. The present study investigated how the coexistence of UGT1A1*6 and UGT1A1"28 may be able to predict toxicities induced by irinotecan in 105 of the patients, and searched for other relevant risk factors.

\section{Materials and methods}

Genetic testing of patients. A total of 2,093 patients with cancer who underwent chemotherapy with irinotecan were recruited from 15 hospitals in Shandong, China (listed in Table I), regardless of diseases and treatment regimens, and were tested for the UGT1A1 genotype between May 2011 and September 2015. The median age of the patients was 58 years (range, 24-89 years). There were 1,414 male and 679 female patients.

UGT1A1 genotyping assay. Genomic DNA was extracted from $2 \mathrm{ml}$ of peripheral blood using a QIAamp Blood kit (Qiagen $\mathrm{GmbH}$, Hilden, Germany). One promoter variant (TA indel) and one exon 1 variant [211G $>A(G 71 R)]$ were genotyped. The TA indel was genotyped as previously described (20). Alleles with 6 and 7 TA repeats are reported as $\mathrm{TA}_{\mathrm{n}}$, and genotypes are assigned based on the number of TA repeats in each allele (including 6/6,6/7 and 7/7).

Genotyping for UGT1A1*6 (211G $>A)$ was performed using the ReverTra Ace qPCR RT kit (cat. no. FSQ-101; Toyobo Co., Ltd., Osaka, Japan). The RT reaction and PCR steps were performed as previously described (21). The primer sequences obtained from Primer Bank (https://pga.mgh.harvard. edu/primerbank/) were 5'-CTCCACCTTCTTTATCTC-3' (forward) and 5'-GCATAGCAGAGTCCTTTT-3' (reverse). Each experiment was repeated three times.

Toxicity studies of irinotecan. Eligibility criteria were as follows: Histologically confirmed diagnosis of the particular tumor type for which irinotecan is indicated; a performance status of 70-90 on the Karnofsky Performance Status (KPS) scale (22); an age of between 22 and 78 years; a predicted life expectancy of at least 3 months; a wash-out period of 12 months after previous irinotecan treatment; adequate base-line organ functions, defined as a total white blood cell count $\geq 3.5 \times 10^{9} / 1$, an absolute neutrophil count $\geq 1.5 \times 10^{9} / 1$, a platelet count $\geq 100 \times 10^{9} / 1$, a hemoglobin level $\geq 90 \mathrm{~g} / 1$, creatinine clearance $>65 \mathrm{ml} / \mathrm{min}$ (according to the Cockcroft formula), alanine transaminase and aspartate transaminase levels $<2.0$ times the upper limit of normal and a total serum bilirubin level $<1.25$ times the upper limit of normal. Exclusion criteria were as follows: Serious infectious diseases or other severe complications, or any other medical problems severe enough to prevent compliance with the protocol. None of the patients were receiving drugs known to interact with irinotecan or to affect the expression and/or function of proteins relevant to irinotecan disposition.

The present study was approved by the Ethics Committees of Qilu Hospital of Shandong University (Shandong, China). Peripheral blood samples were obtained from the patients subsequent to obtaining written informed consent.

Treatment. The present study used two different regimens in this group of patients. Regimen A consisted of irinotecan treatment alone (300-350 mg $/ \mathrm{m}^{2}$ infused for $45 \mathrm{~min}$ intravenously every 3 weeks). Regimen B consisted of irinotecan treatment with antitumor platinum drugs (irinotecan, $250 \mathrm{mg} / \mathrm{m}^{2}$ iv D1; DDP, $25 \mathrm{mg} / \mathrm{m}^{2}$ iv D1-D3, every 3 weeks), or with fluorouracil and derivatives, including 5-fluorouracil (irinotecan, $180 \mathrm{mg} / \mathrm{m}^{2}$ iv D1; $\mathrm{LV}, 400 \mathrm{mg} / \mathrm{m}^{2}$ iv D1; $5-\mathrm{Fu}, 400 \mathrm{mg} / \mathrm{m}^{2}$ iv bolus D1, then $1,200 \mathrm{mg} / \mathrm{m}^{2} \sim 46-48 \mathrm{~h}$, every 2 weeks), capecitabine (capecitabine $1,000 \mathrm{mg} / \mathrm{m}^{2}$,po,bid,D1-14; irinotecan, $250 \mathrm{mg} / \mathrm{m}^{2}$ iv D1, every 3 weeks) and S-1 (irinotecan, $180 \mathrm{mg} / \mathrm{m}^{2}$ iv D1; S-1, $60 \mathrm{mg} / \mathrm{m}^{2}$ po,bid,D1-14, every 3 weeks). Patients underwent chemotherapy cycles until severe toxicity or disease progression appeared. In addition, targeted drug delivery may have been combined with these programs.

Pretreatment evaluation and follow up. The pretreatment evaluation consisted of a complete medical history, physical and imaging examinations, and a hematological laboratory examination. Clinical toxicities, hematological changes and physical condition were monitored prior to each cycle of chemotherapy. The safety population included all patients who received at least one dose of study medication and who had at least one post-baseline safety assessment. The Common Terminology Criteria for Adverse Events v4.0 (23) was used to evaluate adverse events. Furthermore, imaging studies using computed tomography or magnetic resonance imaging were performed prior to the beginning of each following cycle, as well as for confirmation 4 weeks after the end of chemotherapy. Clinical evaluations were performed by a pathologist in a blinded manner with respect to the genetic results, and clinical data were managed by the study organizer (XIUWEN WANG, Qilu Hospital of Shandong University).

Statistical analysis. The direct counting method was used to calculate the allele and genotype frequencies. Hardy-Weinberg equilibrium was measured using the $\chi^{2}$ test. Normality of the data was evaluated using the Shapiro-Wilk test. Measurement data were analyzed using a Student's t-test, and the $\chi^{2}$ test or Fisher's exact test was used to evaluate the association of the UGT1A1 genotype with toxicity and other data, one-way analysis of variance to compare more than two groups, followed by Tukey's test. In addition, logistic regression models were used to analyze the risk factors and interference factors. $\mathrm{P}<0.05$ (two-tailed) was considered to indicate a statistically significant difference. All statistical analyses were performed using SPSS 17.0 (SPSS, Inc., Chicago, IL, USA).

\section{Results}

Distribution of UGT1A1 polymorphisms. Between May 2011 and September 2015, 2,093 and 431 (of those 2,093) patients were tested for the UGT1A1*28 and UGT1A1* 6 mutations, 
Table I. Hospitals from which patients were recruited for the present study.

\begin{tabular}{|c|c|c|}
\hline Hospital name & Location & No. of patients \\
\hline Qilu Hospital of Shandong University & Jinan & 189 \\
\hline Shandong Provincial Hospital affiliated to Shandong University & Jinan & 261 \\
\hline Shandong Cancer Hospital & Jinan & 259 \\
\hline Jinan center Hospital & Jinan & 148 \\
\hline Qingdao Municipal Hospital & Qingdao & 241 \\
\hline Qingdao center medical group & Qingdao & 195 \\
\hline Affiliated Hospital of Qingdao University & Qingdao & 190 \\
\hline Affiliated Hospital of Jining Medical University & Jining & 65 \\
\hline Weifang people's Hospital & Weifang & 63 \\
\hline Yantai Yuhuangding Hospital & Yantai & 50 \\
\hline Linyi people's Hospital & Linyi & 59 \\
\hline Others $^{\mathrm{a}}$ & & 373 \\
\hline \multicolumn{3}{|c|}{$\begin{array}{l}\text { aShandong Qianfoshan Hospital (Jinan), Rizhao people's Hospital (Rizhao), Linyi Cancer Hospital (Linyi), Zhucheng People's Hospital } \\
\text { (Zhucheng), Zhangqiu People's Hospital (Zhangqiu), Binzhou Center Hospital (Binzhou), Shandong Province Traditional Chinese Medicine } \\
\text { (Jinan), Laiyang Center Hospital (Laiyang), The Second Hospital Of Shandong University (Jinan), Jinan Military General Hospital (Jinan), } \\
\text { Affiliated Hospital of Taishan Medical University (Taian), Taian Central Hospital (Taian), Jimo People's Hospital (Jimo), Taian Cancer } \\
\text { Prevention and Treatment Hospital (Taian), Yantaishan Hostital (Yantai), Jining No. } 1 \text { People's Hospital (Jining) and Liaocheng No. } 2 \text { People's } \\
\text { Hospital (Liaocheng). }\end{array}$} \\
\hline
\end{tabular}

Table II. Genotype and allele frequencies.

\begin{tabular}{lc}
\hline Polymorphism & Frequencies \\
\hline UGT1A1 28 (-40_-39insTA) & \\
Genotype & 1601 \\
TA $_{6} / \mathrm{TA}_{6}$ & 463 \\
TA $_{6} / \mathrm{TA}_{7}$ & 29 \\
TA $_{7} / \mathrm{TA}_{7}$ & \\
Allele & \\
TA $_{6}$ & 0.876 \\
TA $_{7}$ & 0.124 \\
UGT1A1 $6(211 \mathrm{G}>\mathrm{A})$ & \\
Genotype & \\
GG & 286 \\
GA & 124 \\
AA & 21 \\
Allele & \\
G & 0.807 \\
A & 0.193 \\
\hline
\end{tabular}

respectively (Fig. 1). Allele frequencies are listed in Table II. The $\chi^{2}$ test shows that the two alleles are consistent with the Hardy-Weinberg equilibrium ( $\mathrm{P}=0.49$ for UGT1A1 $28 ; \mathrm{P}=0.12$ for UGT1A1*6).

Role of UGT1A1 in adverse drug reactions (ADRs) of irinotecan and other high risk factors. Subsequent to evaluation, 105 patients treated in the Department of Chemotherapy, Qilu Hospital of Shandong University, were found eligible and included in the final analysis, although for 4 patients, UGT1A1* 6 status was not determined. The baseline characteristics of the patients are summarized in Table III. There were no significant differences in gender, age, KPS, metastatic occurrence, primary tumor site, irinotecan dose intensity or total bilirubin (TBIL) baseline and hemoglobin (HGB) baseline between double wild-types and mutants $(\mathrm{P}>0.05)$.

ADRs. Tolerance to treatment was evaluated at the first cycle (acute toxicity) and at the end of therapy (cumulative toxicity) (Fig. 2). Severe acute toxicity (3-4) of any type was observed in 30 of 105 patients (28.57\%), and in 42 of 105 patients (40\%) during the entire course of chemotherapy. The most frequent severe hematological toxicity was neutropenia, and the predominant non-hematological toxicities were diarrhea and cholinergic syndrome.

Association between genotypes and ADRs. Table IV compares toxic effects between the $\mathrm{TA}_{6} / \mathrm{TA}_{6}$ group and $\mathrm{TA}_{6} / \mathrm{TA}_{7}$ group. The results showed that the UGT1A1*28 allele was often associated with severe hematological toxicity, particularly during the first cycle. The frequency of grade 3-4 leukopenia and neutropenia in the $\mathrm{TA}_{6} / \mathrm{TA}_{7}$ group was higher compared with the $\mathrm{TA}_{6} / \mathrm{TA}_{6}$ group $(\mathrm{P}<0.05)$.

Considering the low frequency of genotype $\mathrm{A} / \mathrm{A}$, the $\mathrm{A} / \mathrm{A}$ and $A / G$ genotypes were designated as the mutant type for UGT1A1* 6 to study the association between UGT1A1*6 and irinotecan ADRs. It was found that the patients with the mutant type were more susceptible compared with wild-type patients to severe diarrhea and total ADR $(\mathrm{P}=0.030$ and $\mathrm{P}=0.072$, respectively, in the first cycle; $\mathrm{P}=0.043$ and $\mathrm{P}=0.038$, respectively, during the entire course of chemotherapy). The frequency of grade 3-4 neutropenia in the mutant group was higher than that in the wild-type group $(\mathrm{P}=0.003)$. In addition, 
Table III. Patient characteristics and UGT1A1 status.

\begin{tabular}{|c|c|c|c|c|}
\hline Characteristic & Total $(n=103)$ & Wild-type $\left(n=53^{a}\right)$ & Mutant $(\mathrm{n}=50)$ & P-value \\
\hline Gender, $\mathrm{n}$ & & & & 0.554 \\
\hline Male & 65 & 32 & 33 & \\
\hline Female & 38 & 21 & 17 & \\
\hline Age, years $($ mean $\pm S D)$ & & $54.81 \pm 10.77$ & $57.50 \pm 10.81$ & 0.209 \\
\hline $\mathrm{KPS}, \mathrm{n}$ & & & & 0.195 \\
\hline$<90$ & 76 & 42 & 34 & \\
\hline$\geq 90$ & 27 & 11 & 16 & \\
\hline Metastatic, n & & & Miss $1^{\mathrm{b}}$ & 0.636 \\
\hline No & 9 & 4 & 5 & \\
\hline Yes & 93 & 49 & 44 & \\
\hline Primary tumor sites, $\mathrm{n}$ & & & & 0.391 \\
\hline Lung & 16 & 8 & 8 & \\
\hline Colon & 19 & 12 & 7 & \\
\hline Rectum & 21 & 7 & 14 & \\
\hline Esophagus, stomach & 34 & 19 & 15 & \\
\hline Others & 13 & 7 & 6 & \\
\hline Dose intensity (mean \pm SD) & & $185.94 \pm 37.70$ & $182.58 \pm 40.00$ & 0.661 \\
\hline $\mathrm{TBIL}_{\text {baseline }}, \mu \mathrm{mol} / \mathrm{l}($ mean $\pm \mathrm{SD})$ & & $11.64 \pm 8.86$ & $13.50 \pm 16.78$ & 0.478 \\
\hline $\mathrm{HGB}_{\text {baseline }}, \mathrm{g} / \mathrm{l}($ mean $\pm \mathrm{SD})$ & & $118.78 \pm 17.78$ & $124.02 \pm 21.19$ & 0.177 \\
\hline
\end{tabular}

a2 patients with UGT1A1*28 wild-type were excluded from this analysis as genotyping for UGT1A1* 6 was not performed. ${ }^{\mathrm{b}} \mathrm{A}$ patient was excluded from this analysis as the metastatic information was not clear. HGB, hemoglobin; TBIL, total bilirubin; SD, standard deviation; KPS, Karnofsky Performance Status.
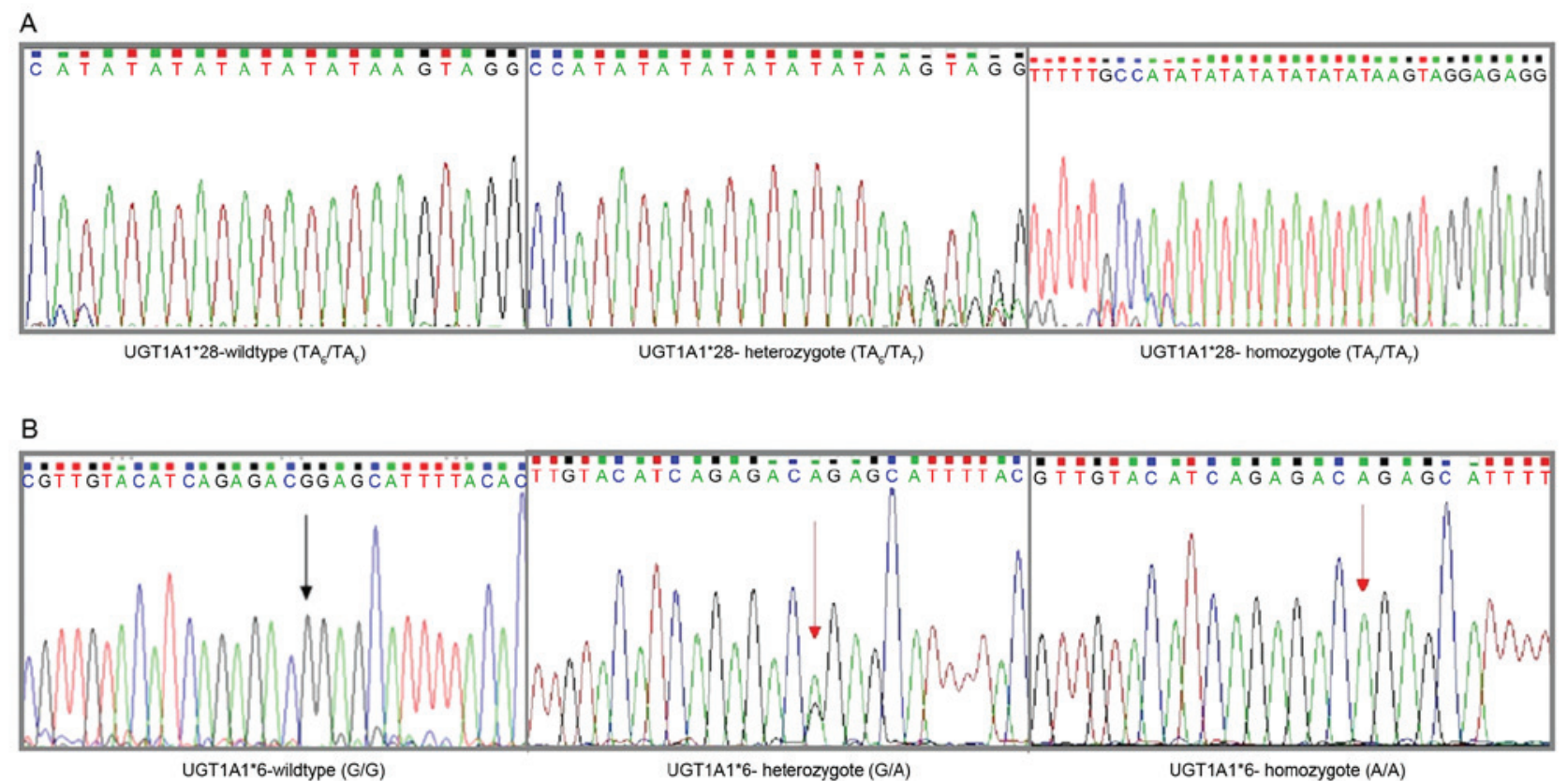

Figure 1. Sequencing results for different UGT1A1*6/28 genotypes. (A) UGT1A1*28 genotype: $\mathrm{TA}_{6} / \mathrm{TA}_{6}$ genotype (left), $\mathrm{TA}_{6} / \mathrm{TA}_{7}$ genotype (middle), $\mathrm{TA}_{7} / \mathrm{TA}_{7}$ genotype (right). (B) UGT1A1*6 genotype: G/G genotype (left), G/A genotype (middle), A/A genotype (right). UGT1A1, uridine diphosphate glucuronosyltransferase $1 \mathrm{~A}$.

the risk of grade 1-4 leukopenia, neutropenia and diarrhea was higher in the $\mathrm{UGT} 1 \mathrm{~A} 11^{*} 6$ carriers compared with wild-type patients $(\mathrm{P}<0.05$; Table V).
TBIL and genotype. In the association between TBIL level and UGT1A1 genotype, neither TBIL $_{\text {baseline }}$ nor TBIL $_{\max }$ showed any significant difference between groups $(\mathrm{P}>0.05)$. In addition, 

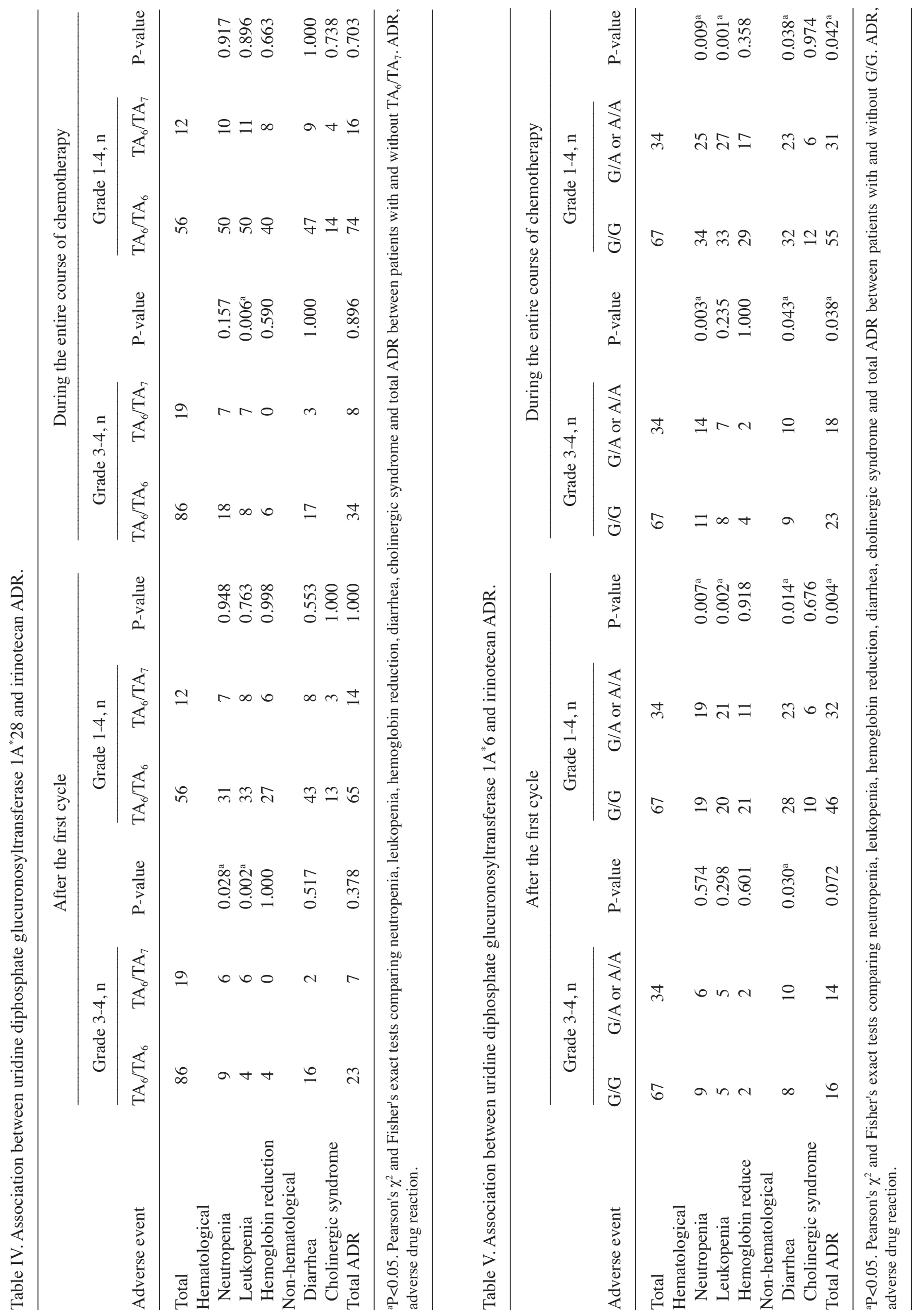
Table VI. Univariate logistic regression analysis for ADRs.

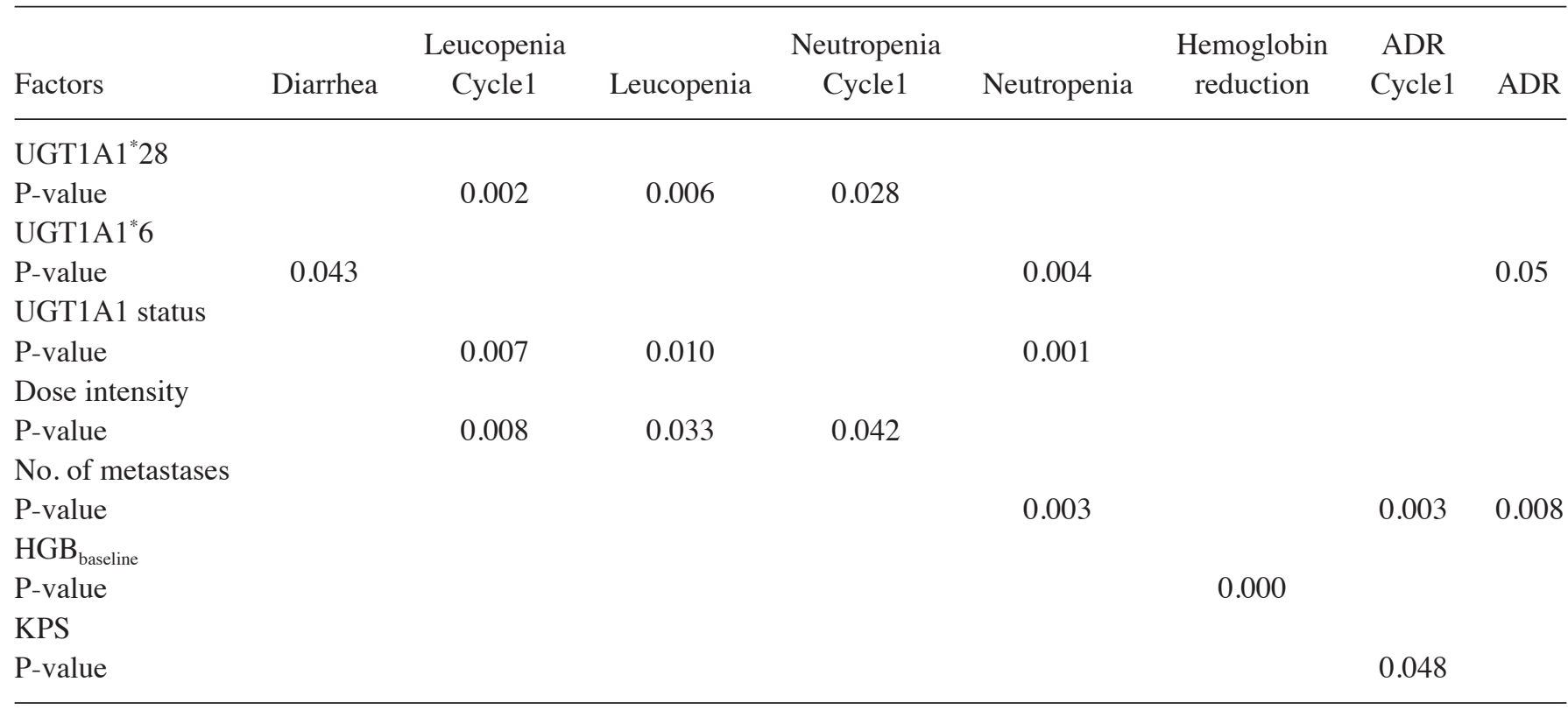

Pearson's $\chi^{2}$ and Fisher's exact tests, and Student's t-test were used to compare clinical factors and all kinds of ADR.

A

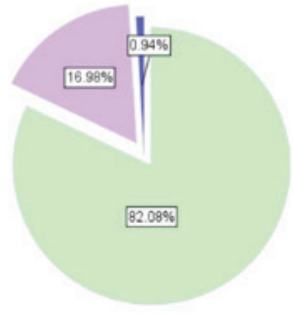

E

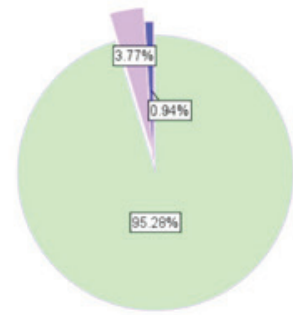

I

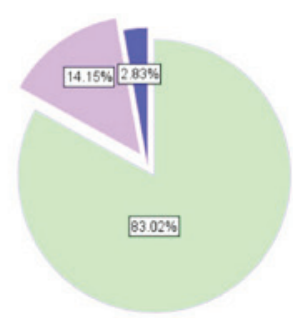

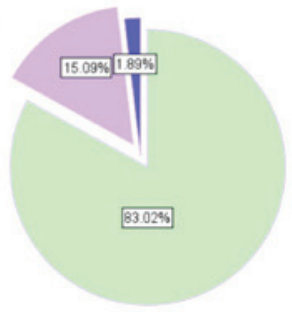

$\mathrm{F}$

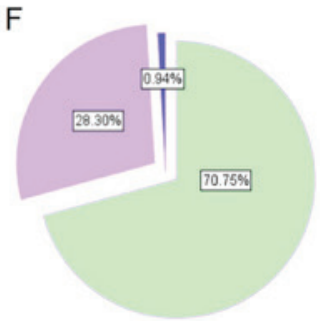

$J$

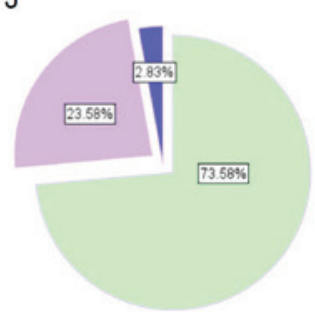

C

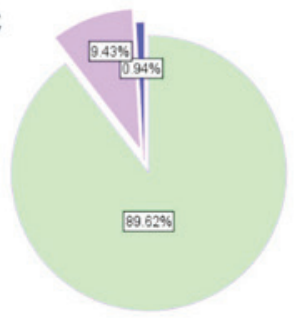

G

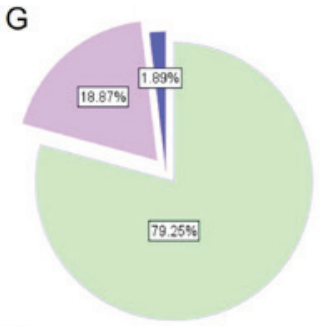

K

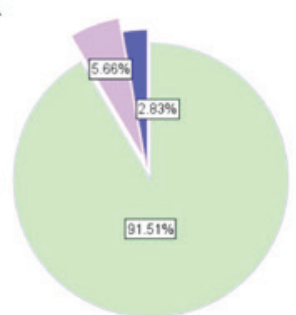

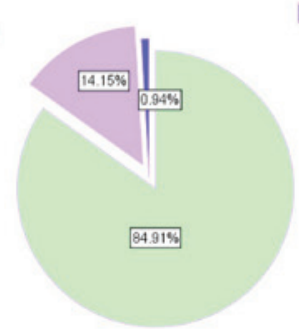

$\mathrm{H}$

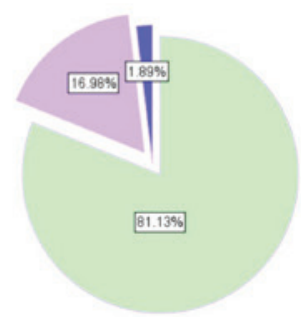

$\mathrm{L}$

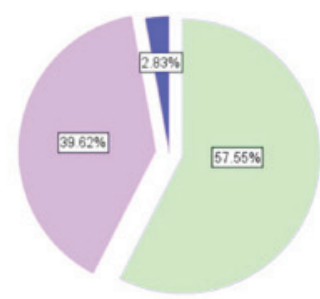

Figure 2. Incidence of adverse reactions. Diarrhea, cholinergic syndrome, leukopenia, neutropenia, reduced hemoglobin and total adverse reactions (A-F) after the first cycle and (G-L) during the entire course of chemotherapy, respectively. 0 2: Mild adverse reactions; 3 4: Severe adverse reactions.

the TBIL level in the patients did not change significantly following treatment when analyzing each genotype separately ( $P>0.05$; data not shown).
Univariate and multivariate analysis of high-risk variables in irinotecan toxicity. To identify the variables of potential predictive significance in irinotecan toxicity, univariate and 
multivariate analyses were performed using the logistic regression model to compare the impact of genotypes and other clinical pathological parameters on the prediction of irinotecan toxicity. Univariate analysis showed that UGT1A1*28, UGT1A1*6, UGT1A1 status, irinotecan dose intensity, occurrence of metastasis, $\mathrm{HGB}_{\text {baseline }}$ and KPS were significant high risk factors for diarrhea, leucopenia, neutropenia, reduced hemoglobin and ADR (Table VI). Multivariate analysis determined that: i) UGT1A1*6 genotype status and total ADR were independent predictors of severe diarrhea $(\mathrm{P}<0.05)$; ii) patients who carry the UGT1A1*28 mutant genotype were more susceptible to severe leucopenia in the first cycle, but the difference was not statistically significant $(\mathrm{P}>0.05)$; iii) the hazard ratio of severe neutropenia in patients with UGT1A1 mutations was 5.859 times that of wild-type patients; iv) with increasing number of metastases, the incidence of severe neutropenia and ADRs was also increased; v) the relative risk in patients with a KPS $<90$ to suffer adverse reactions in cycle 1 was 2.837 times greater than that of patients with a KPS $\geq 90$; vi) irinotecan dose intensity and $\mathrm{HGB}_{\text {baseline }}$ were independent high-risk factors for leucopenia and reduced hemoglobin, separately (Table VII).

\section{Discussion}

The genetic association with irinotecan-related toxicity apparently differs among distinct ethnic populations (24). There is a considerable difference in UGT1A1 genetic polymorphisms among genetically distinct populations; the allele frequency of UGT1A $1{ }^{*} 28$ is higher in Caucasian and African individuals $(0.12-0.27)(25,26)$ compared with Asian individuals ( 0.12) (27). The UGT1A $1^{*} 6$ allele has been identified only in the Asian population (0.13-0.15 for UGT1A1*6) (16). To the best of our knowledge, the present study is the first large-scale study to evaluate the distribution of the UGT1A1 polymorphism in Shandong Province, China. The distribution of UGT1A1 genotypes in the present study was comparable to that of previous studies $(16,26-27)$.

The role of the UGT1A1*28 allele with regard to the toxicity of irinotecan varies greatly between Asian and Caucasian individuals (16). In previous studies, the UGT1A1*28 homozygote has been suggested to associate with neutropenia only in Caucasian individuals (28-31). A meta-analysis by Hoskins et al (24) identified that for irinotecan-induced severe neutropenia, the predictive role of the UGT1A $1^{*} 28$ genotype increased with an increasing dose of irinotecan. Another meta-analysis (32) showed an association between the UGT1A1*28/*28 genotype and an increased risk of neutropenia, at low doses, as well as at medium or high doses of irinotecan. However, a more contentious issue is whether the UGT1A1*28 gene polymorphism can predict severe diarrhea. Marcuello et al (33) found a marked association between severe diarrhea $(\mathrm{P}=0.005)$ and asthenia $(\mathrm{P}=0.03)$, and patients with the heterozygous and homozygous UGT1A1*28 genotypes. In a meta-analysis, patients with a UGT1A1*28/*28 genotype exhibited a higher risk of severe diarrhea at medium and high irinotecan doses (34). However, Stewart et al (31) and the FOCUS trial (35) challenged the aforementioned conclusions, supporting that UGT1A1 genotyping is not a useful prognostic indicator of severe toxicity for patients treated with this irinotecan dosage and schedule. The present study found a significant association between the UGT1A1*28 genotype groups and grade 3-4 hematological toxicity, but not diarrhea, however, this was not confirmed by logistic analysis.

In view of the distribution of the UGT1A $1{ }^{*} 6$ allele in different ethnicities, a previous study by Okuyama et al (36), which focused on Asian individuals, found that homozygosity for UGT1A1 ${ }^{*} 28$ or UGT1A $1^{*} 6$ and double heterozygosity for UGT1A $1 * 28$ and UGT1A1*6 were significantly associated with severe neutropenia $(\mathrm{P}<0.001)$. Sunakawa et al $(37) \mathrm{did}$ not observe any toxic effects that were associated with the UGT1A $1{ }^{*} 1 / * 6$ or UGT1A1*1/*28 genotypes. In the present study, it was found that the incidence of grade 3-4 diarrhea in patients with mutations (A/A and G/A) was much higher than that in wild-type $(\mathrm{G} / \mathrm{G})$ patients. Severe diarrhea and ADR was associated with the UGT1A1*6 genotype in a multiple logistic regression analysis, which also supported this conclusion.

Marcuello et al (33) found that differences in the mean levels of bilirubin among the three genotypes were significant pre- and post-chemotherapy. Bilirubin levels increased significantly when chemotherapy was initiated in $\mathrm{TA}_{6 / 6}$ and $\mathrm{TA}_{6 / 7}$ patients. Stewart et al (31) showed that patients with $\mathrm{TA}_{7 / 7}$ genotype had a statistically greater baseline TBIL compared with patients with the $\mathrm{TA}_{6 / 6}$ or $\mathrm{TA}_{6 / 7}$ genotype. Baseline bilirubin level has also been reported to be associated with severe neutropenia $(12,38)$. In the present study, neither $\mathrm{TBIL}_{\text {baseline }}$ nor $\mathrm{TBIL}_{\text {after max }}$ showed any significant difference between groups. In addition, the TBIL level in patients did not change significantly following treatment when analyzing each genotype separately.

Irinotecan-based genomic studies are no longer restricted to UGT1A1, but also to other genes involved throughout the irinotecan-based metabolic process (28,39-41). Glimelius et al (28) suggested that the ATP-binding cassette sub-family B member 1 gene polymorphism (P-glycoprotein) can predict early adverse reactions. The ATP-binding cassette subfamily $\mathrm{C}$ member 2 (multi-drug resistance protein 2) gene polymorphism was found to be associated with severe diarrhea $(39,40)$. In addition, UGT1 A7 $3 /^{*} 3$ is also known to be associated with early severe neutropenia $(4,21)$. However, it remains unclear whether the UGT1A1 gene polymorphism can be used as a predictor in irinotecan-based toxicity, and the present results provide a novel platform for directing this research.

The present study has certain limitations, including the fact that it is hypothesis generated, due to the retrospective design, and that a relatively small study group was used. Our hypothesis should be confirmed by prospective studies involving larger numbers of patients being performed, and these studies should be aimed at determining whether genotype-adjusted irinotecan dosages could assist in establishing a well-tolerated dose, as well as an effective dose for the tumor response in patients with different genotypes.

In summary, the present data indicated that the UGT1A1*28 and UGT1A1*6 genotypes were significantly associated with severe toxicity, which is an additional supplement to previous studies. Common toxicities can be managed during chemotherapy. However, the clinical implication appears to be marginal. Together, the results of the present and previous studies suggest that genetic testing for the UGT1A $1^{*} 6$ 


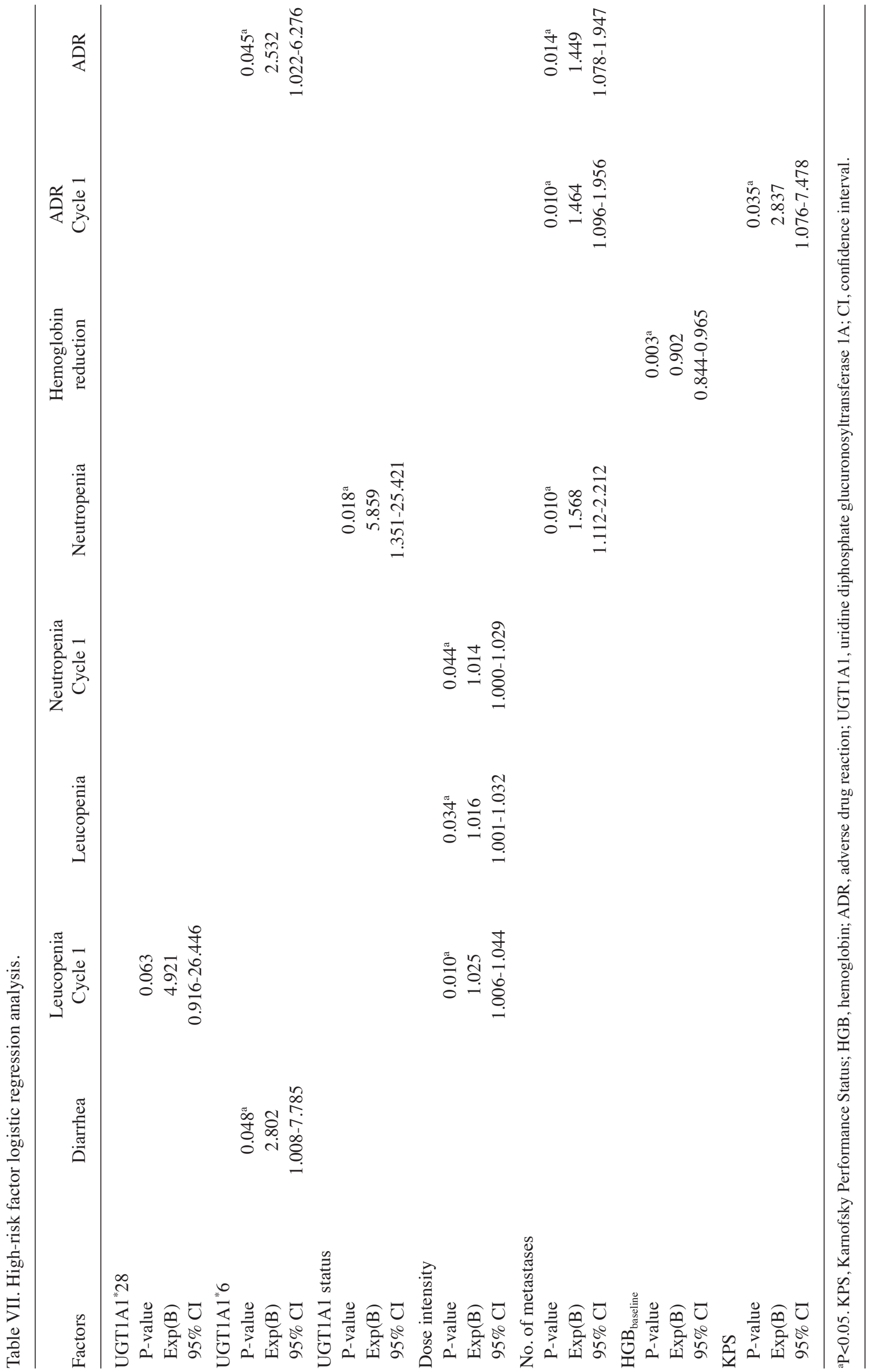


polymorphism may be of use to predict toxicity in patients with cancer who receive irinotecan.

\section{Acknowledgements}

The present study was supported by the Wu Jieping Medical Foundation (grant no. 320.6700.1148) and the National Natural Fund Project (grant no. 81372530).

\section{References}

1. Douillard JY, Cunningham D, Roth AD, Navarro M, James RD, Karasek P, Jandik P, Iveson T, Carmichael J, Alakl M, et al: Irinotecan combined with fluorouracil compared with fluorouracil alone as first-line treatment for metastatic colorectal cancer: A multicentre randomised trial. Lancet 355: 1041-1047, 2000.

2. Tournigand C, André T, Achille E, Lledo G, Flesh M, Mery-Mignard D, Quinaux E, Couteau C, Buyse M, Ganem G, et al: FOLFIRI followed by FOLFOX6 or the reverse sequence in advanced colorectal cancer: A randomized GERCOR study. J Clin Oncol 22: 229-237, 2004.

3. Akie K, Oizumi S, Ogura S, Shinagawa N, Kikuchi E, Fukumoto S, Harada M, Kinoshita I, Kojima T, Harada T, et al: Phase II study of irinotecan plus S-1 combination for previously untreated advanced non-small cell lung cancer: Hokkaido Lung Cancer Clinical Study Group Trial (HOT) 0601. Oncology 81: 84-90, 2011.

4. Takakura S, Takano M, Takahashi F, Saito T, Aoki D, Inaba N, Noda K, Sugiyama T and Ochiai K; Japanese Gynecologic Oncology Group: Randomized phase II trial of paclitaxel plus carboplatin therapy versus irinotecan plus cisplatin therapy as first-line chemotherapy for clear cell adenocarcinoma of the ovary: A JGOG study. Int J Gynecol Cancer 20: 240-247, 2010.

5. Luo HY, Wang ZQ, Wang FH, Qiu MZ, Teng KY, Ruan DY, $\mathrm{He}$ YJ, Li YH and Xu RH: Phase 2 study of capecitabine and irinotecan combination chemotherapy (modified XELIRI regimen) in patients with advanced gastric cancer. Am J Clin Oncol 34: 555-560, 2011.

6. Glimelius B: Benefit-risk assessment of irinotecan in advanced colorectal cancer. Drug Saf 28: 417-433, 2005.

7. Smith NF, Figg WD and Sparreboom A: Pharmacogenetics of irinotecan metabolism and transport: An update. Toxicol In Vitro 20: 163-175, 2006.

8. Mathijssen RH, van Alphen RJ, Verweij J, Loos WJ, Nooter K, Stoter G and Sparreboom A: Clinical pharmacokinetics and metabolism of irinotecan (CPT-11). Clin Cancer Res 7: 2182-2194, 2001

9. Iyer L, Das S, Janisch L, Wen M, Ramírez J, Karrison T, Fleming GF, Vokes EE, Schilsky RL and Ratain MJ: UGT1A1*28 polymorphism as a determinant of irinotecan disposition and toxicity. Pharmacogenomics J 2: 43-47, 2002.

10. Innocenti F and Ratain MJ: 'Irinogenetics' and UGT1A: From genotypes to haplotypes. Clin Pharmacol Ther 75: 495-500, 2004.

11. Perera MA, Innocenti F and Ratain MJ: Pharmacogenetic testing for uridine diphosphate glucuronosyltransferase $1 \mathrm{~A} 1$ polymorphisms: Are we there yet? Pharmacotherapy 28: 755-768, 2008.

12. Ramchandani RP, Wang Y, Booth BP, Ibrahim A, Johnson JR, Rahman A, Mehta M, Innocenti F, Ratain MJ and Gobburu JV: The role of SN-38 exposure, UGT1A $1 * 28$ polymorphism and baseline bilirubin level in predicting severe irinotecan toxicity. J Clin Pharmacol 47: 78-86, 2007.

13. Deeken JF, Slack R and Marshall JL: Irinotecan and uridine diphosphate glucuronosyltransferase 1A1 pharmacogenetics: To test or not to test, that is the question. Cancer 113: 1502-1510, 2008.

14. Ando Y, Saka H, Ando M, Sawa T, Muro K, Ueoka H, Yokoyama A, Saitoh S, Shimokata K and Hasegawa Y: Polymorphisms of UDP-glucuronosyltransferase gene and irinotecan toxicity: A pharmacogenetic analysis. Cancer Res 60: 6921-6926, 2000

15. Han JY, Lim HS, Shin ES, Yoo YK, Park YH, Lee JE, Jang IJ, Lee DH and Lee JS: Comprehensive analysis of UGT1A polymorphisms predictive for pharmacokinetics and treatment outcome in patients with non-small-cell lung cancer treated with irinotecan and cisplatin. J Clin Oncol 24: 2237-2244, 2006.
16. Jada SR, Lim R, Wong CI, Shu X, Lee SC, Zhou Q, Goh BC and Chowbay B: Role of UGT1A1*6, UGT1A1*28 and ABCG2 c. $421 \mathrm{C}>\mathrm{A}$ polymorphisms in irinotecan-induced neutropenia in Asian cancer patients. Cancer Sci 98: 1461-1467, 2007.

17. Onoue M, Terada T, Kobayashi M, Katsura T, Matsumoto S, Yanagihara K, Nishimura T, Kanai M, Teramukai S, Shimizu A, et al: UGT1A1*6 polymorphism is most predictive of severe neutropenia induced by irinotecan in Japanese cancer patients. Int J Clin Oncol 14: 136-142, 2009.

18. Yamamoto N, Takahashi T, Kunikane H, Masuda N, Eguchi K, Shibuya M, Takeda Y, Isobe H, Ogura T, Yokoyama A and Watanabe K: Phase I/II pharmacokinetic and pharmacogenomic study of UGT1A1 polymorphism in elderly patients with advanced non-small cell lung cancer treated with irinotecan. Clin Pharmacol Ther 85: 149-154, 2009.

19. Lu YY, Huang XE, Wu XY, Cao J, Liu J, Wang L and Xiang J: Clinical observations on associations between the UGT1A1 genotype and severe toxicity of irinotecan. Asian Pac J Cancer Prev 15: 3335-3341, 2014

20. Te HS, Schiano TD, Das S, Kuan SF, DasGupta K, Conjeevaram HS and Baker AL: Donor liver uridine diphosphate (UDP)-glucuronosyltransferase-1A1 deficiency causing Gilbert's syndrome in liver transplant recipients. Transplantation 69: 1882-1886, 2000

21. Wang Y, Shen L, Xu N, Wang JW, Jiao SC, Liu ZY and Xu JM: UGT1A1 predicts outcome in colorectal cancer treated with irinotecan and fluorouracil. World J Gastroenterol 18: 6635-6644, 2012.

22. Friendlander AH and Ettinger RL: Karnofsky performance status scale. Spec Care Dentist 29: 147-148, 2009.

23. CTCAE. Cancer Therapy Evaluation Program: Common Terminology Criteria for Adverse Events, Version 4.0. DCTD, NCI, NIH, DHHS, 2010.

24. Hoskins JM, Goldberg RM, Qu P, Ibrahim JG and McLeod HL: UGT1A $1 * 28$ genotype and irinotecan-induced neutropenia: Dose matters. J Natl Cancer Inst 99: 1290-1295, 2007.

25. Yong WP, Innocenti F and Ratain MJ: The role of pharmacogenetics in cancer therapeutics. Br J Clin Pharmacol 62: 35-46, 2006.

26. Premawardhena A, Fisher CA, Liu YT, Verma IC, de Silva S, Arambepola M, Clegg JB and Weatherall DJ: The global distribution of length polymorphisms of the promoters of the glucuronosyltransferase 1 gene (UGT1A1): Hematologic and evolutionary implications. Blood Cells Mol Dis 31: 98-101, 2003.

27. Zhang A, Xing Q, Qin S, Du J, Wang L, Yu L, Li X, Xu L, Xu M, Feng $\mathrm{G}$ and $\mathrm{He} \mathrm{L}$ : Intra-ethnic differences in genetic variants of the UGT-glucuronosyltransferase 1A1 gene in Chinese populations. Pharmacogenomics J 7: 333-338, 2007.

28. Glimelius B, Garmo H, Berglund A, Fredriksson LA, Berglund M, Kohnke H, Byström P, Sørbye H and Wadelius M: Prediction of irinotecan and 5-fluorouracil toxicity and response in patients with advanced colorectal cancer. Pharmacogenomics J 11: 61-71, 2011.

29. McLeod HL, Sargent DJ, Marsh S, Green EM, King CR, Fuchs CS, Ramanathan RK, Williamson SK, Findlay BP, Thibodeau SN, et al: Pharmacogenetic predictors of adverse events and response to chemotherapy in metastatic colorectal cancer: Results from North American Gastrointestinal Intergroup Trial N9741. J Clin Oncol 28: 3227-3233, 2010.

30. Shulman K, Cohen I, Barnett-Griness O, Kuten A, Gruber SB, Lejbkowicz F and Rennert G: Clinical implications of UGT1A $1 * 28$ genotype testing in colorectal cancer patients. Cancer 117: 3156-3162, 2011.

31. Stewart CF, Panetta JC, O'Shaughnessy MA, Throm SL, Fraga $\mathrm{CH}$, Owens T, Liu T, Billups C, Rodriguez-Galindo C, Gajjar A, et al: UGT1A1 promoter genotype correlates with SN-38 pharmacokinetics, but not severe toxicity in patients receiving low-dose irinotecan. J Clin Oncol 25: 2594-2600, 2007.

32. Hu ZY, Yu Q, Pei Q and Guo C: Dose-dependent association between UGT1A1*28 genotype and irinotecan-induced neutropenia: Low doses also increase risk. Clin Cancer Res 16: 3832-3842, 2010.

33. Marcuello E, Altés A, Menoyo A, Del Rio E, Gómez-Pardo M and Baiget M: UGT1A1 gene variations and irinotecan treatment in patients with metastatic colorectal cancer. Br J Cancer 91: 678-682, 2004.

34. Hu ZY, Yu Q and Zhao YS: Dose-dependent association between UGT1A $1 * 28$ polymorphism and irinotecan-induced diarrhoea: A meta-analysis. Eur J Cancer 46: 1856-1865, 2010. 
35. Braun MS, Richman SD, Thompson L, Daly CL, Meade AM, Adlard JW, Allan JM, Parmar MK, Quirke P and Seymour MT: Association of molecular markers with toxicity outcomes in a randomized trial of chemotherapy for advanced colorectal cancer: The FOCUS trial. J Clin Oncol 27: 5519-5528, 2009.

36. Okuyama Y, Hazama S, Nozawa H, Kobayashi M, Takahashi K, Fujikawa K, Kato T, Nagata N, Kimura H, Oba K, et al: Prospective phase II study of FOLFIRI for mCRC in Japan, including the analysis of UGT1A1 28/6 polymorphisms. Jpn J Clin Oncol 41: 477-482, 2011.

37. Sunakawa Y, Ichikawa W, Fujita K, Nagashima F, Ishida H, Yamashita K, Mizuno K, Miwa K, Kawara K, Akiyama Y, et al: UGT1A $1 * 1 / * 28$ and $* 1 / * 6$ genotypes have no effects on the efficacy and toxicity of FOLFIRI in Japanese patients with advanced colorectal cancer. Cancer Chemother Pharmacol 68: 279-284, 2011.

38. Meyerhardt JA, Kwok A, Ratain MJ, McGovren JP and Fuchs CS Relationship of baseline serum bilirubin to efficacy and toxicity of single-agent irinotecan in patients with metastatic colorectal cancer. J Clin Oncol 22: 1439-1446, 2004.
39. Han JY,Lim HS, Shin ES, Yoo YK, Park YH,Lee JE, Kim HT and Lee JS: Influence of the organic anion-transporting polypeptide 1B1 (OATP1B1) polymorphisms on irinotecan-pharmacokinetics and clinical outcome of patients with advanced non-small cell lung cancer. Lung Cancer 59: 69-75, 2008.

40. de Jong FA, Scott-Horton TJ, Kroetz DL, McLeod HL, Friberg LE, Mathijssen RH, Verweij J, Marsh S and Sparreboom A: Irinotecan-induced diarrhea: Functional significance of the polymorphic ABCC2 transporter protein. Clin Pharmacol Ther 81: 42-49, 2007.

41. Lankisch TO, Schulz C, Zwingers T, Erichsen TJ, Manns MP, Heinemann V and Strassburg CP: Gilbert's Syndrome and irinotecan toxicity: Combination with UDP-glucuronosyltransferase 1A7 variants increases risk. Cancer Epidemiol Biomarkers Prev 17: 695-701, 2008. 